\title{
Methodology of the Evaluation of "Komuniti Sihat Pembina Negara-Plus" (KOSPEN Plus) Programme among Workers in Malaysia (Phase 1)
}

\author{
Lim Kuang Kuay ${ }^{1 *}$, Mohd Shaiful Azlan Kassim², Hasimah Ismail ${ }^{3}$, Abdul Aziz \\ Harith $^{4}$, Chan Ying Ying ${ }^{5}$, Zalilah Abdullah ${ }^{6}$, Low Lee Lan $^{7}$, Munirah Ismail ${ }^{8}$, Mohd \\ Azahadi Omar' \\ 1,2,3,4,5 Institute for Public Health, National Institutes of Health, Ministry of Health, Malaysia \\ ${ }^{6,7}$ Institute for Health System Research, National Institute of Health, Ministry of Health, Malaysia \\ ${ }^{8}$ Institute for Health Management, National Institute of Health, Ministry of Health, Malaysia \\ ${ }^{9}$ Data Repository Centre, National Institute of Health, Ministry of Health, Malaysia \\ limkk@moh.gov.my
}

\begin{abstract}
In 2016, the Ministry of Health (MOH) Malaysia has introduced a new programme to prevent and reduce the occurrence of non-communicable diseases (NCDS) as well as related risk factors in the work place environment known as KOSPEN Plus. This study aims to evaluate the awareness and perception of KOSPEN Plus programme after 3 years of implementation in MOH health facilities, government and private agencies in Malaysia. A cross sectional study design was employed in the study. All health facilities, government and private agencies which had implemented KOSPEN Plus programme between 2016 and 31 December 2018 in all states in Malaysia were invited to participate in the study. The computer assisted self-interview (CASI) using the pre-tested selfadministered questionnaire in the electronic form was used. A total of 362 health facilities, government and private agencies which had implemented KOSPEN Plus programme was involved in this study. Overall, more than $80 \%$ of facilities/agencies in this study implemented six out of the eight scopes of KOSPEN Plus. The survey was planned with the goal of obtaining data to support the Ministry of Health in reviewing the present health activities related to workers health in Malaysia and make necessary improvement to the programme.
\end{abstract}

Keywords: KOSPEN Plus; non-communicable diseases; workers; Malaysia

\section{Introduction}

Worldwide, non-communicable diseases (NCDs) continue to be an important public health problem and have begun to receive promising political and social attention from heads of state and ministries of health (Sandeep \& Usnish, 2020; Roura \& Arulkumaran, 2015). The burden of NCDs not only affects high-income nations, as approximately $80 \%$ of premature NCDs deaths occur in low- and middle-income countries (Catherine et al., 2016; Murray et al., 2012). The unhealthy trend will threaten the progress towards the target of the Millennium Development Goals of the United Nations (WHO, 2011) and the affected countries healthcare delivery systems will face enormous pressure to provide quality care to patients with NCDs and their various complications (WHO, 2012). In 2011, the United Nation High-Level Meeting on non-communicable diseases (NCDs) had urged member states to reduce mortality from four major NCDs (cardiovascular diseases, cancers, diabetes, and chronic respiratory diseases) among people aged 30-70 years by $25 \%$ between 2010 and 2025 (WHO, 2013a). The shift of burden from predominantly communicable diseases (CDCs) to NCDs was due to changes in demographic, environmental and the economy of the countries (Samira \& Soma, 2010). A few epidemiological studies in different parts of the country at different time period have demonstrated rising trends of NCDs and NCD risk factors (Prakash et al., 2016). 
According to World Health Organization (2013b), more than 36 million people die annually from chronic diseases, such as cardiovascular diseases, cancers, chronic respiratory diseases and diabetes. Furthermore, it is postulated that this mortality impact will increase to 55 million by 2030. NCDs affect all age groups and the risk factors are mainly due to modifiable behaviours, such as unhealthy diet, inadequate physical activity, tobacco and alcohol use (Krishnan et al., 2010). There were increasing evidence regarding the link between lifestyle risk factors and NCDs (WHO, 2011; Berra et al, 2017) and addressing this lifestyle factors has the potential to reduce the burden of chronic disease to the health system and increase quality-of-life in the individual (Sagner et al., 2014). The future burden of NCDs will not be reversed unless inequities in lifestyle habits and health care access within country borders are remedied (Murray et al., 2012).

In Malaysia, the prevalence of NCD and NCD risk factors have risen substantially in the last two decades (Abrahams-Gessel et al, 2015; Navindra et al., 2018). NCDs are considered the leading causes of death and cardiovascular diseases are responsible for most of the NCD deaths, followed by cancer, respiratory diseases, and diabetes (Feisul et al., 2014). These four groups of diseases account for about $73 \%$ of NCD deaths with $35 \%$ of them comprising deaths of individuals aged less than 60 years (WHO, 2012; Feisul et al., 2014). The Malaysian National Health and Morbidity Survey (NHMS) in 2011 (Azahadi et al., 2019) and 2015 (Feisul et al., 2014) revealed an increasing trend of NCD and NCD risk factors in the adult population. An estimated $35 \%$ of deaths occured in individuals aged less than 60 years, which were mainly the working population 2011 (Azahadi et al., 2019). Despite all the efforts that have been undertaken by local health authorities towards further improving the health status of the population and expanding the scope of NCD prevention and control, the prevalence of NCD and NCD risk factors continues to rise (IPH, 2015; IPH 2011). In view of the increasing trend of NCDs in Malaysia, the Ministry of Health has responded by developing and implementing a new nation-wide programme known as The NCD Prevention- 1Malaysia (NCDP-1M) in 2010 (Abrahams-Gessel et al., 2015; MOH, 2010). In 2013, the ministry has taken a big step by embarking on a nationwide community based intervention program namely Komuniti Sihat Perkasa Negara (KOSPEN). This initiative brings the NCD prevention and control program to the community through trained health volunteers, who will function as health agent of change or health enablers that introduce and facilitate healthy living practices amongst their respective community members (MOH, 2015; Farnaza et al., 2017). Briefly, This programme emphasized on a combination of population or community-based approach with an individually-focused intervention for risk reduction. The principle of community-based action not only targets the community by bringing about behavioural changes, it also includes empowering the community, by encouraging them to act as an agent of change and prompting them to use their own resources for action. In addition, this broad strategy comprises of raising the community's awareness by changing risk perception, providing simple tools, technologies and lifestyle choices as well as facilitating the adoption process of appropriate options by the community members (Lim et al., 2015; Hasimah et al., 2016).

Workplace is also an integral part of the community and the workers spend as much as half of their waking hours at work (Sherry et al., 2017). There is evidence that work such as long work hours, overtime, exposure to shift work, high job demands, and low job control contributes to the development of NCDs (Tu et al., 2010; Sparling 2010). Recognising the increasing trend of NCD and NCD risk factors among workers in Malaysia (IPH, 2016; MOH, 2020), the Ministry of Health (MOH) Malaysia has introduced a new programme to prevent and reduce the occurrence of NCDs as well as related risk factors in the workplace environment known as KOSPEN Plus (Healthy Community Empowers the Nation - Plus) in 2016 (MOH, 2018). Briefly, KOSPEN Plus is an intervention programme initiative between Ministry of Health Malaysia (MOH) and other interested agencies such as health facilities, other government and private agencies. 
Briefly, KOSPEN Plus is an intervention programme initiative between Ministry of Health Malaysia $(\mathrm{MOH})$ and other interested agencies such as health facilities, other government and private agencies. This initiative is a transformation of the public health service in ensuring workers participation in public health programmes.

KOSPEN Plus aims to reduce the occurrence of NCD as well as related risk factors and to increase healthy behaviors including: a) healthy eating, b) smoke free, c) active living, d) weight management, e) health screening, f) healthy mind, g) healthy work place and h) prevention and reducing harmful use of alcohol (IPH, 2015). The programme targets workers and environmental changes to support behavioral modification. The main functioning units for KOSPEN Plus are coordinating team who are trained members from each agency. This team acts as health agent of change towards positive behavioural changes among the workers to adopt and practice healthy lifestyles (IPH, 2015). Such interventions are cost-effective, as they can reduce the need for more expensive treatments if introduced early.

Since the KOSPEN Plus programme has been implemented in 2016, no study was done to evaluate this programme. Evaluation of programme is an integral part in any programme implementation and it will serve as an instrument to know if the intended results are being achieved as planned in a particular programme. Therefore, this study aims to evaluate the awareness and perception of KOSPEN Plus programme after 3 years of implementation in $\mathrm{MOH}$ health facilities, government and private agencies in Malaysia between 2016 and 31 December 2018.

\section{Research Methods}

A cross sectional study design was conducted from January to March 2020. All health facilities, government and private agencies which had implemented KOSPEN Plus programme between 2016 and 31 December 2018 in all 16 states in Malaysia were invited to participate in the study. In each state, a Liaison Officer from the State Health Department was identified to assist the investigators in identifying the study respondents from health facilities, government and private agencies. Then, in each health facility or agency, the Liaison Officer approached one of the members of the KOSPEN Plus coordinating committee or occupational safety and health committee (JKKP) as a respondent to participate in the study. The respondent identified can be either a chairman or deputy chairman or other committee members who are familiar with the KOSPEN Plus implemented in the organization. The respondents were KOSPEN Plus coordinating committee members or occupational safety and health committee (JKKP) members, familiar with the KOSPEN Plus implemented in the organization, provided informed consent to participate and not having any conditions which will affect the data collection.

A self-administered questionnaire in the electronic form was used for data collection. The questionnaire was developed by investigators and pre-tested before being administered to the respondents. The questionnaire contained three modules as follows:

i. Respondent and organization information. This module consists of 8 questions to obtain information on the role of respondent in KOSPEN Plus program, facilities or agency background and the date of KOSPEN Plus programme implemented.

ii. Scope of KOSPEN Plus programme. This module consists of 8 questions on the 8 scope of the KOSPEN Plus programme: 1). healthy eating, 2). smoke free, 3). active living, 4). weight management, 5). health screening, 6). healthy mind, 7). healthy work place and 8). prevention and reducing harmful use of alcohol.

iii. Perception toward KOSPEN Plus programme. This module consists of eight questions to obtain information on the perception of the each KOSPEN Plus scope implemented at the facility or agency. 
Computer-Assisted Self-Interview (CASI) approach was employed to obtain data. The survey questionnaire was sent to the respondents' preferred email address. They do not need to sign in a new account in order to fill in the questionnaire/consent form. First, the respondents need to read/understand the information about the survey and provide informed consent by pressing all the "AGREE" and "SEND" buttons located in the "RESPONSE AGREEMENT" section to enable them to answer the survey question. Participation in this survey is free and the survey takes about 20 minutes to answer. The respondents identity were created prior to data collection and the respondents required to enter their answers depending on the types of response required in the questionnaire; such as free text, numeric or alphanumeric answers. After respondents completed the questionnaire, a final button will direct the respondents to submit data to the server. All questions must be answered by the respondents and in order to minimise the unanswer questions, the application will automatically remind the respondents if the related questions are not answered.

Quality control of the survey was done at various stages to ensure data collected was of high-quality. During the data collection, a Liaison Officer from the State Health Department was given responsibility to supervise the whole data collection processes. At the central level, the progress of data collection for all facilities and agencies was monitored by the dedicated officer from the Institute for Public Health (IPH) on a daily basis. Furthermore, all questionnaires submitted to the IPH need to undergo various checks and were verified by a verifier to ensure the validity of the answers.

The study ethical approval was obtained from the Medical and Research Ethics Committee (MREC), Malaysian Ministry of Health (NMRR-18-3965-40445) and permission to conduct the study was obtained from all the health facilities, government and private agencies involved. The research was funded by the Institute for Public Health, Ministry of Health Malaysia. The purpose of the study was explained to the respondents in the electronic consent form and informed consent was obtained from each respondent before the start of the survey. Participation in this survey is entirely voluntary and if they decided to withdraw from the survey midway, they could exit the site freely. No measures will be used to preserve the data they have filled in, and all answered data from them will be destroyed. All the consent forms, personal information and other data collected will be kept for 3 years in a secure filing cabinet and handled in accordance the Ministry of Health data storage regulations and Data Protection Act. This data will be destroyed after 3 years of storage. When publishing or presenting the study results, the respondents' identity will not be revealed without their expressed consent.

In this study, all data processing activities were centralized at Institute for Public Health (IPH). The collected data in the IPH server were coded. Each data was coded accordingly so that no mistake and no irregularities prevailed that might give an incorrect report. The data was printed out to detect any irregularities. SPSS software version 23 (IBM SPSS, Chicago) was used for data analysis. Descriptive statistic was used to illustrate the socio-demographic characteristics of the respondents. Analysis was done according to the objectives of the study. Complex sample analysis procedures were used in the analysis and was carried out at $95 \%$ confidence interval. 


\section{Results and Discussion}

A total of 362 health facilities, government and private agencies which had implemented KOSPEN Plus programme was involved in this study. Overall, more than $80 \%$ of facilities/agencies in this study implemented six out of the eight scopes of KOSPEN Plus. Majority of the scopes implemented by the facilities/agencies was health screening $(95.6 \%, \mathrm{n}=346)$ followed by Healthy mind $(92.0 \%, \mathrm{n}=333)$, active living $(89.2 \%, \mathrm{n}=89.2)$, smoke-free $(88.4 \%, \mathrm{n}=320)$, healthy eating practices $(82.6 \%, \mathrm{n}=299)$ and weight management $(82.0 \%, \mathrm{n}=297)$. The two optional scopes that showed the lowest implementation by the facilities/agencies were healthy work place $(78.8 \%, \mathrm{n}=285)$ and prevention and reducing harmful use of alcohol $(54.7 \%, \mathrm{n}=198)$ (Table 1).

Among health facilities $(n=275)$ which had implemented KOSPEN Plus, majority of facilities implemented health screening $(96.7 \%, \mathrm{n}=266)$ followed by healthy mind $(94.5 \%, \mathrm{n}=260)$, active living $(90.2 \%, \mathrm{n}=248)$ and smoke free $(90.2 \%, \mathrm{n}=248)$, healthy eating practices $(83.3 \%, \mathrm{n}=229)$, weight management $(82.5 \%, \mathrm{n}=227)$ Meanwhile the scopes that were poorly implemented in health facilities were healthy workplace $(79.3 \%, \mathrm{n}$ $=218)$ followed by prevention and reduction of harmful alcohol $(53.3 \%, \mathrm{n}=147)$ (Table $1)$.

Among government agencies $(n=74)$ that were involved to implement the KOSPEN Plus programme, mostly carried out health screening $(90.5 \%, \mathrm{n}=67)$, followed by active living $(86.5 \%, \mathrm{n}=64)$, smoke free $(85.1 \%, \mathrm{n}=63)$, healthy mind $(83.8 \%, \mathrm{n}=62)$, healthy eating practices $(83.8 \%, \mathrm{n}=62)$ and weight management $(81.1 \%, \mathrm{n}=60)$. Programme activities that were lack implemented by agencies and health facilities were similar, such as healthy workplace $(79.7 \%, \mathrm{n}=59)$, followed by prevention and reduction of harmful use of alcohol $(60.8 \%, \mathrm{n}=45)$ (Table 1$)$.

The results revealed that $100 \%(n=30)$ of private agencies were involved and implemented KOSPEN Plus program scope for health screening followed by active living $(84.6 \%, \mathrm{n}=11)$, healthy mind $(84.6 \%, \mathrm{n}=11)$ and weight management $(76.9 \%, \mathrm{n}=10)$. Meanwhile, program activities that are poorly implemented by private agencies were smoke free $(69.2 \%, \mathrm{n}=9)$, followed by healthy workplace $(61.5 \%, \mathrm{n}=8)$, healthy eating practices $(61.5 \%, \mathrm{n}=8)$ and prevention and reduction of harmful use of alcohol $(46.2 \%, \mathrm{n}$ =6) (Table 1). 
Table 1. Implementation of KOSPEN Plus in Health Facilities, Government Agencies and Private Agencies, by Scope

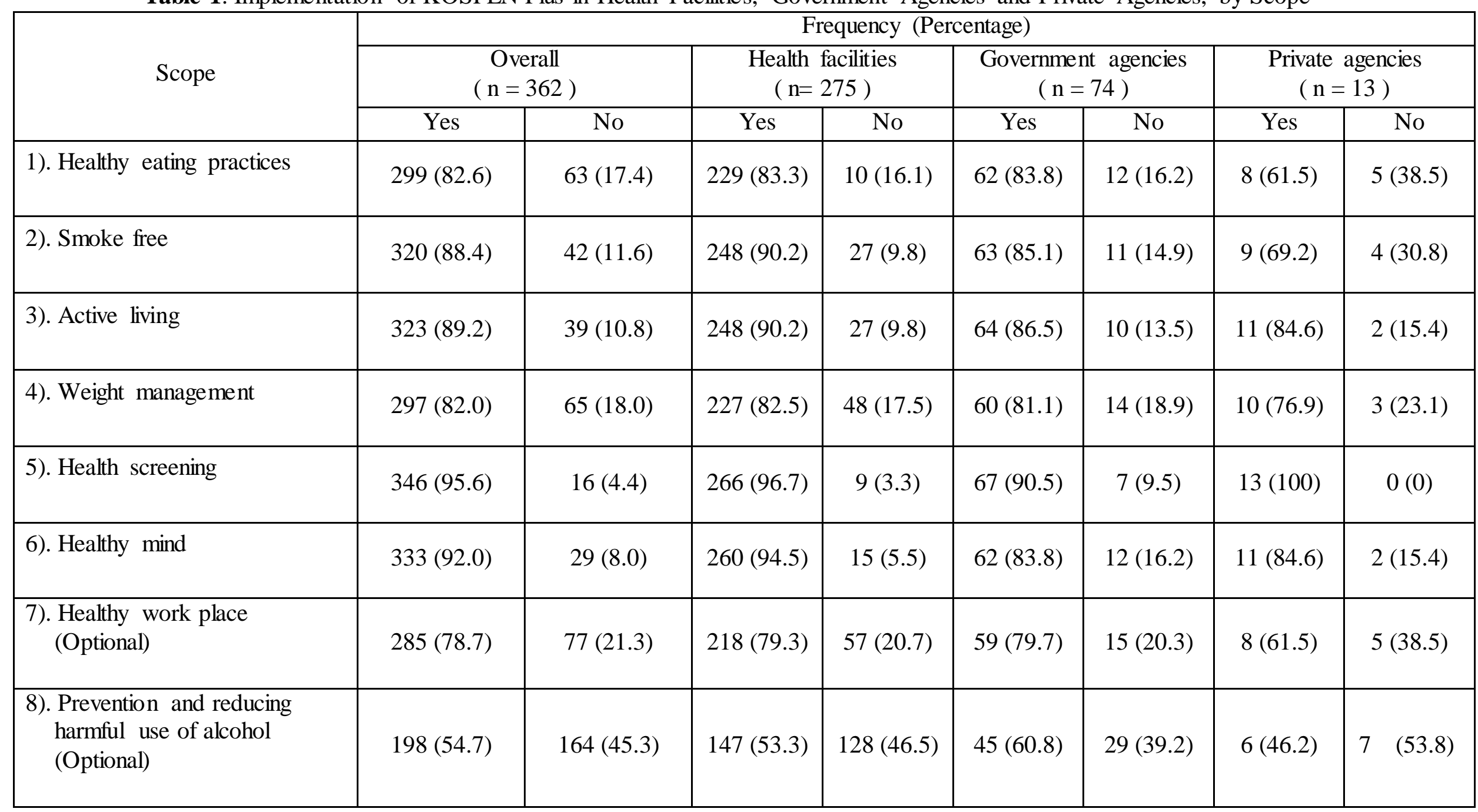




\section{Conclusion}

Overall, KOSPEN Plus programme was very well accepted by the facilities/agencies involved. The successful implementation of KOSPEN Plus programme among workers in Malaysia depends greatly on the commitments from three main groups; Ministry of Health $(\mathrm{MOH})$, the employer and the liaison officer (a group of people appointed by employers that form a functional unit that acts as advocator, promoter and advisor for workers). The $\mathrm{MOH}$ role is to provide technical input and expertise in training, supervision and monitoring of the programme. While, the employer vital role is to ensure sustainability of programme by providing support, commitment, direction, resources forming policies and enforcement of programme implementation. In addition, the liaison officers are responsible in implementing the KOSPEN Plus activities according to the identified scopes. The current survey was planned with the goal of obtaining data to support Ministry of Health in reviewing the present health activities related to workers health in Malaysia and make necessary improvement to the programme. It was hoped that the KOSPEN Plus programme will have a positive impact on health status of workers in Malaysia.

\section{Acknowledgements}

The authors would like to thank the Director-General of Health, Malaysia for his permission to publish this paper and those who were involved in the study.

\section{References}

Sandeep PK, Usnish BM. 2020. Learning from progress: global NCD policy implementation at national level. Lancet Glob Health. 8(1):e4-e5.

Roura LC, Arulkumaran SS. 2015. Facing the noncommunicable disease (NCD) global epidemic e The battle of prevention starts in utero - The FIGO challenge. Best Pract \& Res Clini Obstet Gynaecol. 29:5e14.

Catherine PB, Gregory AR, Andrew EM. 2016. The Global Burden of Disease Study and the Preventable Burden of NCD. Global Heart. 11(4):393-97.

Murray CJ, Vos T, Lozano R, Maghavi M, Flaxman AD, Michaud C, et al. 2012. Disability-adjusted life years (DALYs) for 291 diseases and injuries in 21 regions, 1990-2010: a systematic analysis for the Global Burden of Disease Study 2010. Lancet. 380(9859):2197-223.

World Health Organization (WHO). 2011. Global status report on noncommunicable diseases 2010. Geneva: World Health Organization.

World Health Organization (WHO). 2012. Noncommunicable diseases in the Western Pacific Region: a profile. Geneva: World Health Organization.

World Health Organization (WHO). 2013a. NCD global monitoring framework. [internet]. [cited July 20, 2020]; available from: https://www.who.int/nmh/global_ monitoring_framework/en/.

Samira HH, Soma S. 2010. Burden of non-communicable disease: Global overview. Diabetes Metab Syndr Clin Res Rev. 4:41- 47.

Prakash CN, Raman C, Vivek R, Vidyasagar, Kavinder L. 2016. Epidemiological study of non-communicable diseases (NCD) risk factors in tribal district of Kinnaur, HP: A cross-sectional study. Indian Heart J. 68:655-62.

World Health Organization (WHO). 2013b. Global action plan for the prevention and control of non-communicable diseases 2013-2020. Geneva: World Health Organization.

Krishnan A, Ekowati R, Baridalyne N, Kusumawardani N, Suhardi, Kapoor SK, Leowski 
J. 2010. Evaluation of community-based interventions for non-communicable diseases: experiences from India and Indonesia. Health Promotion Int. 26(3):276-89.

Berra K,ranklin B, Jennings C. 2017. Community-Based healthy living interventions. Prog in Cardiovasc Dis. 59:430-34.

Sagner M, Katz D, Egger G, Lianov L, Schulz KH, Braman M, et al. 2014. Lifestyle medicine potential for reversing a world of chronic disease epidemics: from cell to community. Int J Clin Pract. 68(11):1289-92.

Abrahams-Gessel S, Denmany CA, Montanoz CM, Gaziano TA, Levittk N, RiveraAndradez A, et al. 2015. Training and supervision of community health workers conducting population-based, noninvasive screening for CVD in LMIC. Global Heart. 10:39-44.

Navindra KS, Hayati K, Rosliza AM. 2018. Factors Associated with Knowledge of Healthy Community,Empowers Nation (KOSPEN) and its Implementation among Community Health Volunteers in Kulim District, 2017. Mal J Med Health Sci. 14(2):7-16.

Feisul IM, Zainal AO, Omar M, Kamaliah MN, Noraryana H, Rotina AB, et al. 2014. Addressing non-communicable diseases in Malaysia: an integrative process of systems and community. BMC Public Health. 14(Suppl 2):S4.

Azahadi O, Shubash SG, Mohamad Fuad MA, Yi Yi K, Chandrika J, S. Maria A,et al. 2019. Cause-specific mortality estimates for Malaysia in 2013: results from a national sample verification study using medical record review and verbal autopsy. BMC Public Health. 19:10.

Institute for Public Health (IPH). 2015. National health and morbidity survey 2015 (NHMS 2015). Vol. II: non-communicable diseases, risk factors \& other health problems. Kuala Lumpur, Institute for Public Health.

Institute for Public Health (IPH). 2011. National Health and Morbidity Survey 2011 (NHMS 2011). Vol. II: Non-Communicable Diseases. Kuala Lumpur: Ministry of Health Malaysia.

Ministry of Health (MOH). 2010. National strategic plan for non-communicable disease. Kuala Lumpur: Ministry of Health.

Ministry of Health, Malaysia (MOH). 2015. Health Facts 2015 [internet]. [cited 20 July 2020]. available from: http://vlib.moh.gov.my/cms/documentstorage/com. tms.cms.document.Document_ ef876440 -a0188549-82a26f00-e6a36876/KKM_ HEALTH_FACTS_2015.pdf.

Farnaza A, Anis SR, Maryam HD, Jamaiyah H, Suraya AR, Sharmini S, et al. 2017. Feasibility of Implementing Chronic Care Model in the Malaysian Public Primary Care Setting. Med J Malaysia. 72(2):106-112.

Lim KH, Fadhli, Omar M, Rosnah MN, M. Nazaruddin B, Sumarni MG et al. 2015. Technical Report Evaluation of effectiveness of implementation of "komuniti sihat perkasa negara" (KOSPEN) programme in Malaysia- phase 1. Kuala Lumpur: Institute for Public Health.

Hasimah Ismail, Dr Rosnah Ramly, Dr Tahir Aris, Dr Muhammad Fadhli Mohd Yusoff et al. 2016. Technical Report Evaluation of the implementation of "komuniti sihat perkasa negara” programme in Malaysia (KOSPEN). Kuala Lumpur: Institute for Public Health.

Sherry OP, Amy Mc, Ross A, Lawrence C. 2017. Healthy Living Medicine in the Workplace: More Work to Do". Progress in Cardiovasc Diseases. 59:440-47.

Tu HT, Boukus ER, Cohen GR. 2010. Workplace clinics: a sign of growing employer interest in wellness". Res Brief. 17:1-16.

Sparling PB. 2010. Worksite health promotion: principles, resources, and challenges". Prev Chronic Diseases. 7(1):A25. 
Institute for Public Health. 2016. A study on determination of cause of deaths in Malaysia. Kuala Lumpur: Institute for Public Health.

Ministry of Health (MOH). 2020. Malaysian Health at a Glance 2018. Ministry of Health Malaysia.

Ministry of Health (MOH). 2018. KOSPEN Plus programme guideline: Intervention non communicable diseases risk factor at work place. Ministry of Health Malaysia. 